\title{
PREPRINTS
}

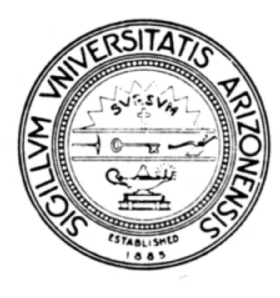

$\mathrm{OF}$ THE

\author{
STEWARD OBSERVATORY \\ THE UNIVERSITY OF ARIZONA \\ TUCSON, ARIZONA
}

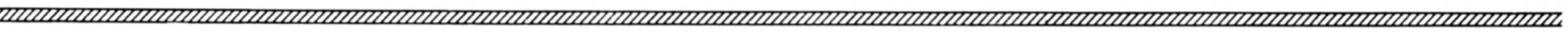

NO. 25

POLARIZATION OF RADIO SOURCES

II

FARADAY EFFECT IN THE CASE OF QUASITRANSVERSE PROPAGATION

by

A. G. Pacholczylk

T. L. Swihart 


\section{POLARIZATION OF RADIO SOURCES}

II

FARADAY EFFECT IN THE CASE OF QUASITRANSVERSE PROPAGATION

by

A. G. Pacholczyk

and

T. L. Swihart

Steward Observatory, University of Arizona 


\begin{abstract}
Under the conditions of quasitransverse propagation of electromagnetic waves in a magnetoionic plasma, the effect analogous to Faraday rotation in the quasilongitudinal case (which we will call Faraday pulsation) can produce a large elliptical polarization of originally linearly polarized radiation. Therefore, the presence of elliptical polarization in the radiation cannot serve as a means for distinguishing between a synchrotron mechanism with high energy electrons and any other type of emission process in radio sources in which conditions suggesting quasitransverse propagation (large magnetic fields) may take place. The Faraday pulsation has a wavelength dependence through which it can be identified.
\end{abstract}




\section{INTRODUCTION}

The existence of high intensity magnetic fields in certain radio sources is now considered plausible. For example, fields of the order of $10^{2}-10^{3} \mathrm{G}$ have been suggested for quasars and Seyfert galaxies (cf. Burbidge and Burbidge 1967; Pacholczyk and Weymann 1968; Sturrock and Feldman 1968), while fields in the range of $10^{10}-10^{16} \mathrm{G}$ are commonly suggested for neutron stars and pulsars (Woltjer 1964; Gold 1968; Pacini 1968). In sufficiently large magnetic fields the quasitransverse (QT) approximation for the propagation of radiation in a plasma should be used rather than the quasilongitudinal (QL) approximation. The QT approximation for a collisionless plasma is valid if the following inequality is satisfied:

$$
\frac{\sin ^{4} \theta}{4 \omega^{2} \theta} \frac{\omega_{H}^{2}}{\omega^{2}} \gg\left(1-\frac{\omega_{0}^{2}}{\omega^{2}}\right)^{2} \text {, }
$$

(see, e.g., Ratcliffe 1962). In the above, $\theta$ is the angle between the magnetic field and the direction of propagation; $\omega_{\mathrm{H}}=\mathrm{eH} / \mathrm{mc}$ is the electron gyrofrequency in the magnetic field $\mathrm{H} ; \omega_{\mathrm{O}}=\left(4 \pi \mathrm{e}^{2} \mathrm{~N} / \mathrm{mc}\right)^{1 / 2}$ is the plasma frequency corresponding to electron density $\mathrm{N}$; and $\omega$ is the radiation frequency. Relation (1) will be violated for sufficiently small values of the angle $\theta$, but high intensity magnetic fields insure that it will be satisfied for a significant range of $\theta$.

In the QL approximation the ordinary and extraordinary rays are circularly polarized, and the difference in propagation constants between the two rays leads to the well-known effect of Faraday rotation. In the QT approximation, however, the ordinary and extraordinary rays are plane polarized. The changing phase difference between the rays in this case leads to a quite different effect, which we call Faraday pulsation. The 
main features of Faraday pulsation were briefly noted by Mass (1966). In the present paper we incorporate the effect into the equation of transfer, discuss solutions for some special cases, and point out some important observational consequences.

\section{THE EQUATIONS OF TRANSFER}

As in Pacholczyk and Swihart (1967; hereafter referred to as Paper I), $(1,2)$ and $(a, b)$ represent two orthogonal coordinate systems in the plane normal to the direction of propagation--the former being fixed at each point by the local magnetic field and the latter being fixed with respect to the observer. Direction 2 is along the component of $\overrightarrow{\mathrm{H}}$ normal to the direction of propagation, and $x$ is the angle between $a$ and 2 . The $(1,2)$ and $(a, b)$ systems coincide if $x=\frac{\pi}{2}$.

In the $(a, b)$ system the radiation field is specified by the four Stokes parameters $I_{a}, I_{b}, U$ and $V$. The equations of transfer for these quantities are given in terms of the absorption and emission coefficients and $\Delta \mathrm{k}$, the difference between the propagation constants for the extraordinary ray and the ordinary ray. In the QT approximation one has, using the notation of Paper I:

$$
\begin{aligned}
& \mathrm{dI} a / d s=I_{a}\left[-\kappa_{1} \sin ^{4} x-\kappa_{2} \cos ^{4} x-1 / 4\left(\kappa_{1}+\kappa_{2}\right) \sin ^{2} 2 x\right] \\
& +U\left[1 / 4\left(k_{1}-k_{2}\right) \sin 2 x\right]+\varepsilon_{1} \sin ^{2} x+\varepsilon_{2} \cos ^{2} x-1 / 2 \Delta \mathrm{k} v \sin 2 x \text {, } \\
& \mathrm{dI}_{\mathrm{b}} / \mathrm{ds}=\mathrm{I}_{\mathrm{b}}\left[-k_{1} \cos ^{4} \mathrm{x}-\kappa_{2} \sin ^{4} x-1 / 4\left(k_{1}+k_{2}\right) \sin ^{2} 2 x\right] \\
& +U\left[1 / 4\left(k_{1}-k_{2}\right) \sin 2 x\right]^{2}+\varepsilon_{1} \cos ^{2} x+\varepsilon_{2} \sin ^{2} x+1 / 2 \Delta k v \sin 2 x \text {, } \\
& \mathrm{dU} / \mathrm{ds}=\mathrm{I}_{\mathrm{a}}\left[1 / 2\left(\kappa_{1}-\kappa_{2}\right) \sin 2 x\right]+I_{b}\left[1 / 2\left(\kappa_{1}-\kappa_{2}\right) \sin 2 x\right] \\
& -1 / 2\left(\kappa_{1}+k_{2}\right) U-\left(\varepsilon_{1}-\varepsilon_{2}\right) \sin 2 x+\Delta k V \cos 2 x, \\
& \mathrm{dV} / \mathrm{ds}=\Delta \mathrm{k}\left(\mathrm{I}_{\mathrm{a}}-\mathrm{I}_{\mathrm{b}}\right) \sin 2 \mathrm{x}-\Delta \mathrm{k} \mathrm{U} \cos 2 \mathrm{x}-1 / 2\left(\mathrm{k}_{1}+\kappa_{2}\right) \mathrm{V} \text {. }
\end{aligned}
$$


The above equations of transfer describe linearly polarized emission and absorption. For unpolarized absorption, and emission, $\kappa_{1}=\kappa_{2}=\kappa$, $\varepsilon_{1}=\varepsilon_{2}=1 / 2 \varepsilon$. For high energy synchrotron processes, $\varepsilon_{1}$ and $\varepsilon_{2}$ are given, e.g., by Ginzburg and Syrovatskii (1965), and $\kappa_{1}$ and $\kappa_{2}$ are given by equations (2) of Paper I.

The difference between propagation constants $\Delta \mathrm{k}$ is given in the QT approximation by

$$
\Delta \mathrm{k}=\frac{\omega}{\mathrm{c}}\left[1-\left(\frac{\omega^{2}-\omega_{0}^{2}}{\omega^{2}-\omega_{0}^{2} \cos ^{2} \theta}\right) 1 / 2\right],
$$

(see, e.g., Ratcliffe 1962). In the limit $\omega^{2}>>\omega_{0}^{2}$ this equation reduces to the following:

$$
\Delta \mathrm{k}=\frac{\omega_{0}^{2} \sin ^{2} \theta}{2 \omega c}=\frac{2 \pi \mathrm{e}^{2} \mathrm{~N}}{\mathrm{mc} \omega} \sin ^{2} \theta .
$$

Note that, in the QT approximation, $\Delta \mathrm{k}$ does not depend on the strength of the magnetic field, and is proportional to the wavelength.

For a homogeneous medium the angle $x$ in equations (2) is a constant. Choosing $x=\frac{\pi}{2}$ so that the $(1,2)$ and $(a, b)$ systems coincide, one finds that equations (2) reduce to the following:

$$
\begin{aligned}
& \mathrm{dI}_{\mathrm{a}} / \mathrm{ds}=-\kappa_{1} \mathrm{I}_{\mathrm{a}}+\varepsilon_{1}, \quad \mathrm{dI}_{\mathrm{b}} / \mathrm{ds}=-\kappa_{2} \mathrm{I}_{\mathrm{b}}+\varepsilon_{2}, \\
& \mathrm{dU} / \mathrm{ds}=-k \mathrm{U}-\Delta \mathrm{k} \mathrm{V}, \mathrm{dV} / \mathrm{ds}=-\kappa \mathrm{V}+\Delta \mathrm{k} \mathrm{U} .
\end{aligned}
$$

where $k=1 / 2\left(\kappa_{1}+\kappa_{2}\right)$. Thus the only coupling remaining is that between $\mathrm{U}$ and $\mathrm{V}$. The solutions for the latter are:

$$
\begin{aligned}
& \mathrm{U}=\left(\mathrm{U}_{\mathrm{o}} \cos \Delta \mathrm{ks}-\mathrm{V}_{\mathrm{o}} \sin \Delta \mathrm{ks}\right) \mathrm{e}^{-\mathrm{Ks}}, \\
& \mathrm{V}=\left(\mathrm{U}_{\mathrm{o}} \sin \Delta \mathrm{ks}+\mathrm{V}_{\mathrm{o}} \cos \Delta \mathrm{ks}\right) \mathrm{e}^{-\mathrm{Ks}} .
\end{aligned}
$$




\section{FARADAY PULSATION}

The solutions of equations (5) (for simplicity we assume that there is no absorption) indicate that the degree of polarization, $\Pi$, changes from its initial value, $\Pi_{0}$, for $s=0$ to the value $\left(\varepsilon_{1}-\varepsilon_{2}\right) /\left(\varepsilon_{1}+\varepsilon_{2}\right)$ for $s \rightarrow \infty$, and is not influenced by the Faraday effect. The position angle $x$,

$$
\tan 2 x=\frac{U}{I_{1}-I_{2}}=\frac{U_{0} \cos \Delta k s-v_{0} \sin \Delta k s}{I_{10}-I_{20}+\left(\varepsilon_{1}-\varepsilon_{2}\right) s},
$$

and the ellipticity $\beta$,

$$
\sin , 2 \beta=\frac{V}{I_{1}+I_{2}}=\frac{U_{0} i x \Delta k s+V_{0} \sin \Delta k}{I_{10}+I_{20}+\left(\varepsilon_{1}+\varepsilon_{2}\right) s},
$$

are very much influenced by the Faraday effect. For example, for initially linearly polarized radiation (high energy synchrotron radiation, $V_{0}=0$ ), the position angle will oscillate periodically between $\chi_{0}$ and $-\chi_{0}$ if there is no polarized emission within the plasma itself. If there is polarized emission within the plasma, the amplitude of oscillation will gradually decrease from $x_{0}$ to zero with increasing $s$. An originally linearly polarized beam will acquire certain ellipticity through the Faraday effect, the maximum ellipticity ' $\beta$ being equal to $\chi_{0}$ if there is no emission within the plasma. If there is emission, the periodically reached maxima of ellipticity will be decreasing with increasing $s$.

For initially circularly polarized light $\left(I_{10}+I_{20}=V, U_{0}=0\right)$ we have

$$
\tan 2 x=\frac{I_{0}}{\left(\varepsilon_{1}-\varepsilon_{2}\right) s} \operatorname{six} \Delta \mathrm{ks} \text {, }
$$

and

$$
\sin 2 \beta=\frac{I_{0}}{I_{0}+\left(\varepsilon_{1}-\varepsilon_{2}\right) s} \cos \Delta,
$$


When there is no emission within the plasma, the radiation varies between the two extremes of linear and circular polarization periodically, and the position angle stays at $45^{\circ}$ to the field. It was pointed out by Legg and Westfold (1968) that when the energies of synchrotron radiating electrons are relatively low, the radiation will be characterized by a certain amount of elliptical polarization, even if coming from a relatively isotropic distribution of electrons. This amount of elliptical polarization will not necessarily be that observed from such a radio source with a strong field, as the plasma in the source and its vicinity alters the polarization characteristics through the Faraday pulsation effect.

It is seen from the above that Faraday pulsation does not occur for plane polarized radiation if the plane of polarization is either parallel or normal to the magnetic field. Thus synchrotron radiation emitted by high energy electrons in a plasma with a uniform magnetic field will not undergo Faraday pulsation from that plasma, since its plane of polarization is normal to the magnetic field; therefore it is of interest to examine a case in which the field has a variable direction. As an example we consider a plasma of dimension L along the line of sight, homogeneous except that the direction of the magnetic field changes such that $x=\frac{\pi}{2}+\delta s, 0 \leqslant s \leqslant L$, where $\delta$ is a constant. Figure 1 shows the solutions of equations (2) for $\delta=\Delta k=4 \pi / L$, without absorption. The emission is assumed to come from a point source located at $s_{0}$ (the path length within the plasma is then $\mathrm{L}-\mathrm{s}_{\mathrm{o}}$ ), and it is linearly polarized normal to the field at that point; the original degree of polarization is 0.724 . The abscissa in the figure is the source position in units of $2 \pi / \delta$, and the ordinates are the Stokes parameters $Q\left(=I_{a}-I_{b}\right), U$, and $V$ normalized to unit intensity. We see that in this case as well, a substantial circularly 
polarized component is produced by Faraday pulsation for appropriate values of $\mathrm{L}-\mathrm{s}_{\mathrm{O}}$. This simple example shows how a variable polarization, including a variable circular component, can be obtained by having high energy particles injected into the plasma at different points at different times.

We thank Mr. N. D. Lubart for computing the curves in Fig. 1. 


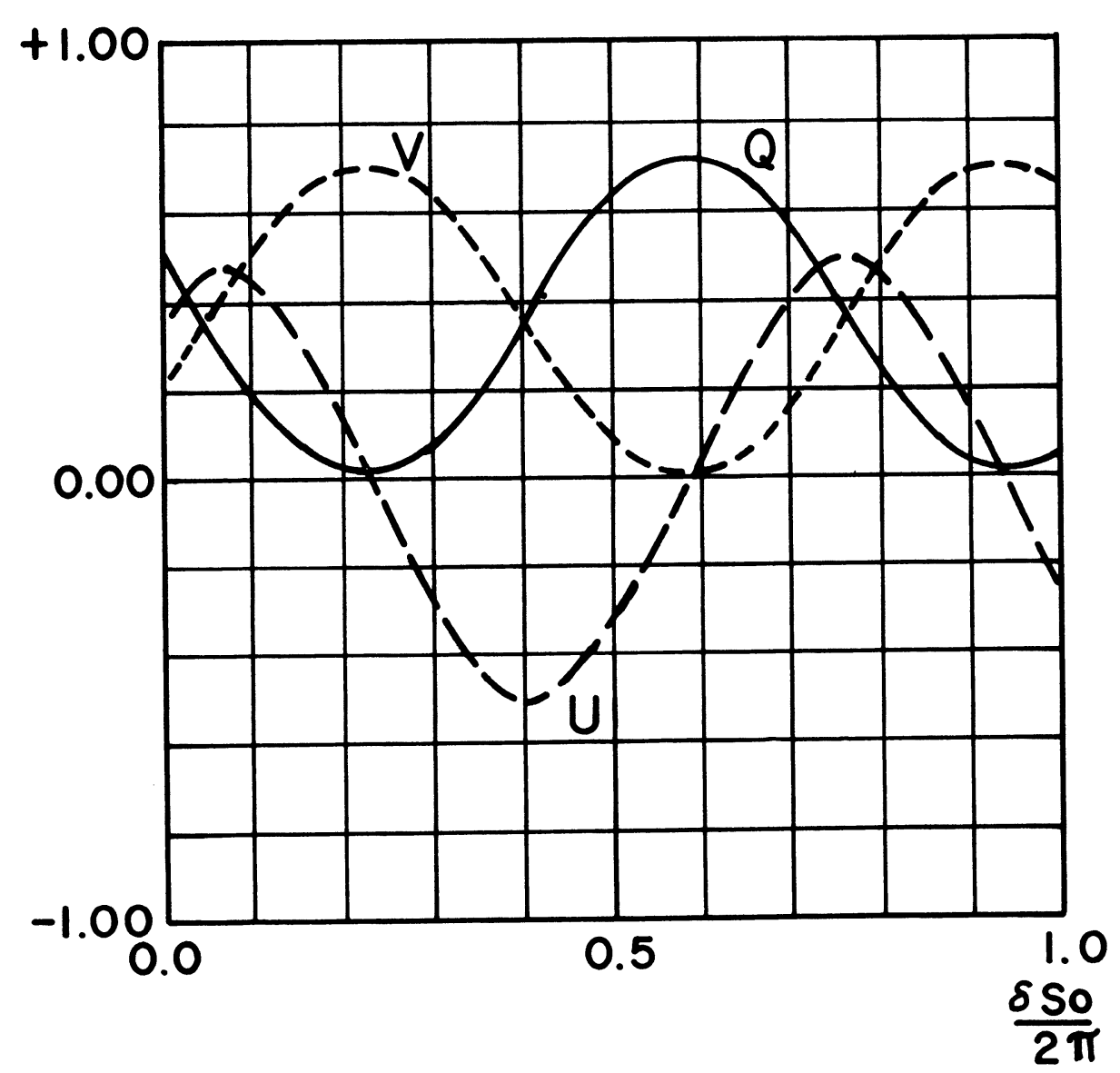

Fig. 1. - The Stokes parameters Q, U, and V, normalized to unit intensity, of the radiation emerging from a plasma of thickness $L=4 \pi / \delta$. The abscissa is the position of the point source in units of $2 \pi / \delta$. The radiation is assumed to have a degree of polarization of $72.4 \%$, which fixes the maximum values of the parameters. 


\section{REFERENCESS}

Burbidge, G., and Burbidge, M. 1967, Quasi-Stellar Objects (San Francisco: Freeman and Co.).

Ginzburg, V. L., and Syrovatskii, S. I. 1965, Ann. Rev. Astron. and Ap., 3, 297 .

Gold, T. 1968, Nature, 218, 731.

Legg, M. P. C., and Westfold, K. C. 1968, Ap. J., 154, 499.

Mass, J. 1966, Radio Science, 1 , 1137.

Pacholczyk, A. G., and Swihart, T. L. 1967, Ap. J., 150, 647.

Pacholczyk, A. G., and Weymann, R. J. 1968, A.J., 73, 870 .

Pacini, F. 1968, Nature, 219, 145 .

Ratcliffe, J. A. 1962, The Magneto-Ionic Theory (Cambridge: University Press).

Sturrock, P. A., and Feldman, P. A. 1968, A.J., 73, 910.

Woltjer, L. 1964, Ap. J., 140, 1309. 